\title{
Time-Domain Anyon Interferometry in Kitaev Honeycomb Spin Liquids and Beyond
}

\author{
Kai Klocke $\odot^{1,2}$ David Aasen ${ }^{3,4}$ Roger S. K. Mong, ${ }^{5,6}$ Eugene A. Demler, ${ }^{7}$ and Jason Alicea ${ }^{1,8}$ \\ ${ }^{1}$ Department of Physics and Institute for Quantum Information and Matter, California Institute of Technology, \\ Pasadena, California 91125, USA \\ ${ }^{2}$ Department of Physics, University of California, Berkeley, California 94720, USA \\ ${ }^{3}$ Kavli Institute for Theoretical Physics, University of California, Santa Barbara, California 93106, USA \\ ${ }^{4}$ Microsoft Quantum, Microsoft Station Q, University of California, Santa Barbara, California 93106-6105, USA \\ ${ }^{5}$ Department of Physics and Astronomy, University of Pittsburgh, Pittsburgh, Pennsylvania 15260, USA \\ ${ }^{6}$ Pittsburgh Quantum Institute, Pittsburgh, Pennsylvania 15260, USA \\ ${ }^{7}$ Department of Physics, Harvard University, 17 Oxford Street, Cambridge, Massachusetts 02138, USA \\ ${ }^{8}$ Walker Burke Institute for Theoretical Physics, California Institute of Technology, Pasadena, California 91125, USA
}

(Received 9 November 2020; accepted 30 March 2021; published 27 April 2021)

\begin{abstract}
Motivated by recent experiments on the Kitaev honeycomb magnet $\alpha-\mathrm{RuCl}_{3}$, we introduce time-domain probes of the edge and quasiparticle content of non-Abelian spin liquids. Our scheme exploits ancillary quantum spins that communicate via time-dependent tunneling of energy into and out of the spin liquid's chiral Majorana edge state. We show that the ancillary-spin dynamics reveals the edge-state velocity and, in suitable geometries, detects individual non-Abelian anyons and emergent fermions via a time-domain counterpart of quantum-Hall anyon interferometry. We anticipate applications to a wide variety of topological phases in solid-state and cold-atoms settings.
\end{abstract}

DOI: 10.1103/PhysRevLett.126.177204

Introduction.-Topologically ordered phases that support non-Abelian anyons-fractionalized quasiparticles exhibiting noncommutative braiding statistics-provide a potential quantum-computing medium with intrinsic fault tolerance [1,2]. To this end, developing single-anyon detection techniques poses a key challenge, both for validating anyonic content and for readout. Earliest efforts centered around the fractional quantum Hall $(\mathrm{FQH})$ state at filling $\nu=5 / 2$ [3], which is now widely believed to realize the non-Abelian Moore-Read state [4] (or one of its cousins [5-7]) [8]. There, electrical anyon interferometry [9-12] enables single-anyon detection and has been explored both at $\nu=5 / 2$ [13] and especially decisively in the Abelian $\nu=1 / 3$ state [14].

More recent experiments spotlight a fundamentally different candidate non-Abelian anyon platform: quantum spin liquids in spin-orbit-coupled Mott insulators governed by variants of Kitaev's honeycomb model [15-17]. In particular, thermal-transport measurements $[18,19]$ on $\alpha-\mathrm{RuCl}_{3}$ [20] suggest that the non-Abelian spin-liquid phase from Kitaev's model emerges upon application of an $\mathcal{O}(10 \mathrm{~T})$ magnetic field (see also Ref. [21]). This putative spin liquid mimics the Moore-Read state, without the charge sector; it supports a chiral Majorana edge state, trivial bosonic excitations (1), emergent fermions $(\psi)$, and "Ising" non-Abelian anyons $(\sigma)$. Crucially, however, detection methods do not directly carry over from the quantumHall problem due to the host system's Mott-insulating character. While several recent works have nevertheless devised electrical spin-liquid probes [22-27], the problem certainly warrants renewed attention.

We introduce a single-anyon detection scheme naturally tailored to a Mott-insulating spin system. Our approach eschews electrical measurements altogether in favor of time-domain probes of ancillary spins dynamically coupled to the spin liquid's chiral Majorana edge state. Timedomain techniques have been profitably employed to study chiral topological phases in various contexts, including detection of edge magnetoplasmons [28], generation of coherent single-electron excitations on quantum-Hall edges [29], and edge-mediated state transfer [30]. In our proposal, an "emitter" ancillary spin shuttles (bosonic) energy via the chiral Majorana edge state toward a downstream "absorber" ancillary spin [Fig. 1(a)]. If the spin liquid contains a constriction, as in Fig. 2, en route the injected energy can splinter such that a fractionalized edge excitation encircles a bulk quasiparticle (of type $\mathbb{1}, \psi$, or $\sigma$ ). Crucially, the probability of energy capture by the absorber spin depends on the bulk quasiparticle type by virtue of nontrivial braiding statistics, thereby enabling single-anyon detection via a time-domain analog of $\mathrm{FQH}$ interferometry. This scheme extends to general Abelian and non-Abelian chiral topological phases and appears particularly well-suited for insulating magnets and cold atoms.

Edge-state interrogation.-We first illustrate how our methods enable time-domain exploration of edge states. Suppose that two ancillary spin- $1 / 2$ degrees of freedom $\boldsymbol{s}_{1,2}$ locally couple to the spin liquid's chiral Majorana edge 


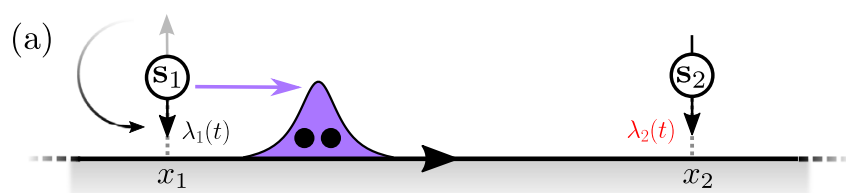

(b)
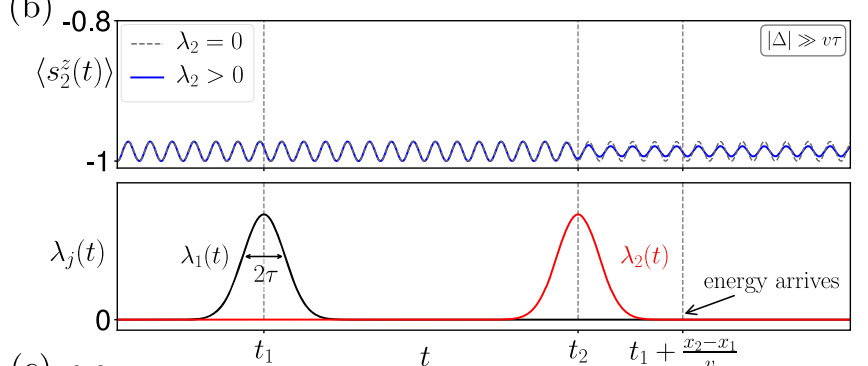

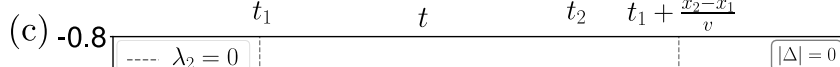
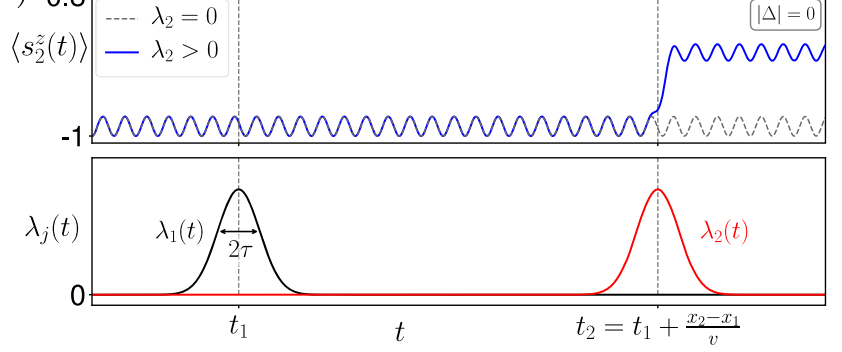

FIG. 1. (a) Ancillary spins $\boldsymbol{s}_{1,2}$ interacting with a chiral Majorana edge state via time-dependent couplings $\lambda_{1,2}(t)$. At time $t=0, s_{1}$ and $s_{2}$ are respectively initialized into excited (up) and ground-state (down) spin configurations. Pulsing $\lambda_{1}(t)$ allows $s_{1}$ to relax, depositing excess energy (purple) into the edge as a pair of Majorana fermions (black dots) that propagate chirally toward $\boldsymbol{s}_{2}$. An "aligned" $\lambda_{2}(t)$ pulse, timed to coincide with the arrival of the energy packet, allows $\boldsymbol{s}_{2}$ to retrieve the incident energy. (b) Time evolution of $\left\langle s_{2}^{z}(t)\right\rangle$ (top panel) for a "misaligned" pulse sequence (bottom panel) such that $\lambda_{2}(t)$ turns on prior to the arrival of energy injected by $s_{1}$. The small suppression of oscillations after the misaligned $\lambda_{2}$ pulse reflects an interplay with spin precession. (c) Same as (b) but for an aligned $\lambda_{2}(t)$ pulse. Energy shuttling from $s_{1}$ to $s_{2}$ mediates a spin flip responsible for the late-time "kick" in $\left\langle s_{2}^{z}(t)\right\rangle$. Parameters for (b),(c): $h_{x} / h_{z}=1 / 6, h_{z} \tau=3$, $\tau /\left(t_{2}-t_{1}\right)=1 / 20, \bar{\lambda}_{1,2} h_{z} / v^{2}=1.275$.

mode at positions $x_{1,2}$ [Fig. 1(a)]. We model the dynamics with a Hamiltonian [31]

$$
H=-i v \int_{x} \gamma \partial_{x} \gamma+\sum_{j=1,2}\left[\boldsymbol{h} \cdot \boldsymbol{s}_{j}+\frac{\lambda_{j}(t)}{2 \pi} s_{j}^{x} T\left(x_{j}\right)\right],
$$

where the Majorana field obeys $\left\{\gamma(x), \gamma\left(x^{\prime}\right)\right\}=\frac{1}{2} \delta\left(x-x^{\prime}\right)$, $s_{j}^{\alpha}$ are Pauli operators acting on the ancillary spins, and $T(x)=-2 \pi i: \gamma \partial_{x} \gamma:$ is the normalized stress-energy tensor for the edge conformal field theory. The first term in Eq. (1) describes the edge kinetic energy with velocity $v$, while the second captures the ancillary-spin Zeeman energy. Throughout we assume for simplicity $h_{z} \gg\left|h_{x}\right|$ and $h_{y}=0$. The third hybridizes the ancillary spins to the edge state via couplings $\lambda_{j}(t)$ that descend from exchange interactions with the non-Abelian spin liquid $[22,30]$; these terms mediate energy shuttling between the ancillary spins by allowing each spin to locally absorb or deposit energy packets consisting of an even number of fermionic edge excitations. We assume that the $\lambda_{j}(t)$ couplings, and hence shuttling of energy, are amenable to real-time control.

Figure 1 sketches the protocol of interest: (i) Start with $\lambda_{1,2}=0$ and prepare an initial state $|\phi(t=0)\rangle=$ $|0\rangle \otimes\left|s_{1}^{z}=\uparrow, s_{2}^{z}=\downarrow\right\rangle$, where $|0\rangle$ describes the vacuum for the Majorana edge mode; note the excess energy $\sim 2 h_{z}$ for $\operatorname{spin} s_{1}$. (ii) Turn on a Gaussian pulse $\lambda_{1}(t)=$ $\bar{\lambda}_{1} e^{-\left(t-t_{1}\right)^{2} /\left(2 \tau^{2}\right)}$ that enables $s_{1}$ to shed energy into the edge, where it propagates chirally toward $s_{2}$ at speed $v$. (iii) Turn on $\lambda_{2}(t)=\bar{\lambda}_{2} e^{-\left(t-t_{2}\right)^{2} /\left(2 \tau^{2}\right)}$. We take $h_{z} \tau \gg 1$ so that the pulses approximately conserve energy [32]. If the duration between pulses satisfies $v\left(t_{2}-t_{1}\right) \approx x_{2}-x_{1}$ (within a tolerance of $v \tau$ ), then energy that $s_{1}$ deposits to the edge arrives coincident with the $\lambda_{2}$ pulse and can thus be absorbed by $s_{2}$. Spin $s_{2}^{z}$ is measured at some time $t>0$.

We compute the expectation value $\left\langle s_{2}^{z}(t)\right\rangle$ perturbatively in $h_{x} / h_{z}$ and $\Lambda_{j} \equiv \bar{\lambda}_{j} h_{z} / v^{2}$ (the dimensionless spin-edge coupling strength at energy scale $h_{z}$ ), assuming $\left(t_{2}-t_{1}\right) \gg \tau$. For details, see Supplemental Material [33]. At measurement times $t \gg t_{2}$, we find

$$
\begin{aligned}
\left\langle s_{2}^{z}(t)\right\rangle \approx & -1+\left(h_{x} / h_{z}\right)^{2} \sin ^{2}\left(h_{z} t\right) \\
& -\frac{1}{12 \sqrt{\pi}} \Lambda_{2}^{2}\left(\frac{h_{x}}{h_{z}}\right)^{2}\left(h_{z} \tau\right) \cos \left(2 h_{z} t\right) \\
& +\frac{1}{36 \pi}\left(\Lambda_{1} \Lambda_{2}\right)^{2}\left(h_{z} \tau\right)^{2} e^{-\frac{\Delta^{2}}{2(v \tau)^{2}}}
\end{aligned}
$$

where $\Delta=v\left(t_{2}-t_{1}\right)-\left(x_{2}-x_{1}\right)$ quantifies the timing mismatch between the pulses. The first line reflects spin precession from the Zeeman field. The second line is independent of pulse timing and originates from processes whereby $h_{x}$ flips $s_{2}^{z}$ from down to up, after which $\lambda_{2}$ mediates a second spin flip. Most importantly, the final line-which depends exponentially on pulse misalignment $\Delta$-is the correction due to energy shuttling from $s_{1}$ to $s_{2}$. Figures 1(b) and 1(c), respectively, display $\left\langle s_{2}^{z}(t)\right\rangle$ for misaligned $(|\Delta| \gg v \tau)$ and aligned $(|\Delta| \approx 0)$ pulses. Observing the additional "kick" visible in the aligned case provides a direct measure of the edge-state velocity $v$. If phase coherence is maintained over the length $x_{2}-x_{1}$, then we recover an additional oscillatory correction due to interference between the energy shuttling and the precession of both spins (see Supplemental Material [33]).

Time-domain anyon interferometry.-Next we revisit the above double-pulse protocol in an interferometer geometry featuring a constriction in the spin liquid [Figs. 2(a),(b)]. We are specifically interested in the energy-shuttling probability when a bulk quasiparticle of type $a=\mathbb{1}, \psi$, or $\sigma$ resides in the enclosed region. At the constriction, bosonic energy packets injected from spin $s_{1}$ can splinter 

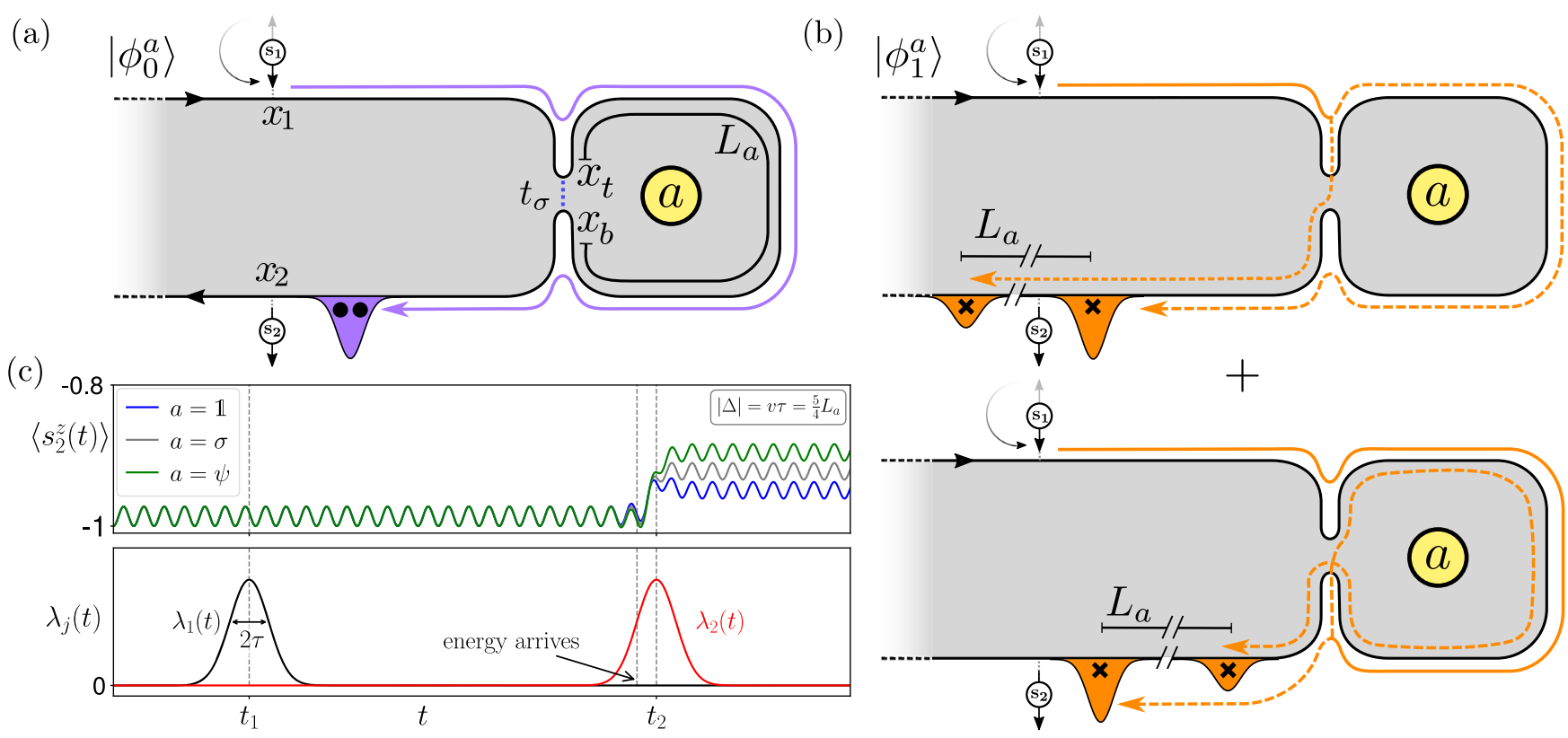

FIG. 2. (a),(b) Interferometer enabling detection of a bulk quasiparticle of type $a=\mathbb{1}, \psi$, or $\sigma$ residing in the enclosed region of length $L_{a}$. The initialization and pulse sequence are the same as in Fig. 1. For the trivial path depicted in (a), $s_{1}$ injects energy that bypasses the constriction and travels the long way toward $s_{2}$. For the two $\mathcal{O}\left(t_{\sigma}\right)$ tunneling paths in (b), the energy instead splinters into Ising anyons (indicated by x's) at the constriction, one of which encircles quasiparticle $a$; the wave packets for the outgoing Ising-anyon edge excitation are separated by $L_{a}$. Interference between these paths yields an $a$-dependent probability for energy absorption by $\boldsymbol{s}_{2}$. (c) Time evolution of $\left\langle s_{2}^{z}(t)\right\rangle$ (top panel) for a partially misaligned $\left(|\Delta|=\frac{5}{4} L_{a}\right) \lambda_{1,2}(t)$ pulse sequence (bottom panel). Crucially, the late-time behavior discriminates the three quasiparticle types. Parameters are $\tilde{t}_{\sigma}=2$ and $v \tau / L_{a}=5 / 4$, with others as given in Fig. 1(c).

into fractionalized edge excitations. We include only the most relevant process wherein Ising anyons tunnel between positions $x_{t}$ and $x_{b}$ across the pinch [34-36]:

$$
H_{\text {tun }}=t_{\sigma} e^{-i \pi h_{\sigma}} \sigma\left(x_{b}\right) \sigma\left(x_{t}\right) \text {. }
$$

Here $\sigma(x)$ is the Ising-anyon field with conformal weight $h_{\sigma}=1 / 16$, and $x_{b}-x_{t} \equiv L_{a}$ defines the path length enclosing quasiparticle $a$. We work in the regime where the dimensionless tunneling parameter $\tilde{t}_{\sigma} \equiv t_{\sigma} L_{a}^{7 / 8} / v$ admits a perturbative treatment [22].

Let $\left|\phi^{a}(t)\right\rangle=\left|\phi_{0}^{a}(t)\right\rangle+\left|\phi_{1}^{a}(t)\right\rangle+\cdots$ denote the system's wave function with $\left|\phi_{j}^{a}(t)\right\rangle$ the $\mathcal{O}\left(\tilde{t}_{\sigma}^{j}\right)$ component. To $\mathcal{O}\left(\tilde{t}_{\sigma}\right)$, we have $\left\langle s_{2}^{z}(t)\right\rangle=f_{0}(t)+f_{\text {int }}^{a}(t)$, where

$$
f_{0}=\left\langle\phi_{0}^{a}\left|s_{2}^{z}\right| \phi_{0}^{a}\right\rangle, \quad f_{\text {int }}^{a}=2 \operatorname{Re}\left\langle\phi_{0}^{a}\left|s_{2}^{z}\right| \phi_{1}^{a}\right\rangle .
$$

In the dominant wave function component, $\left|\phi_{0}^{a}\right\rangle$, energy deposited by $s_{1}$ travels a distance $x_{2}-x_{1}$ the long way around the constriction before reaching $\boldsymbol{s}_{2}$. For $h_{x} \neq 0$, there is also a term in $\left|\phi_{0}^{a}\right\rangle$ involving the precession of both spins. The associated contribution $f_{0}(t)$ to $\left\langle s_{2}^{z}(t)\right\rangle$ from this trivial path is given at late times by Eq. (2). In the subleading wave function component $\left|\phi_{1}^{a}\right\rangle$, energy imparted by $s_{1}$ splinters into two Ising anyons-one of which carries some fraction of the incident energy across the constriction and, crucially, encircles quasiparticle $a$. Two such paths arise [see Fig. 2(b)] depending on whether tunneling happens from above or below the constriction. Energy packets that hop across the constriction travel a distance $\left(x_{2}-x_{1}\right) \pm L_{a}$, while the remainder of the energy travels a distance $x_{2}-x_{1}$, coincident with the trivial path. [We assume that $\boldsymbol{s}_{2}$ sits sufficiently far from the constriction that the tunneled Ising anyon from Fig. 2(b), bottom, completely braids around $a$ before any energy arrives to $x_{2}$.] Interference between the trivial path and the two $\mathcal{O}\left(\tilde{t}_{\sigma}\right)$ paths depends on the enclosed quasiparticle type $a$ and underlies the correction $f_{\text {int }}^{a}$ in Eq. (4).

Non-Abelian statistics kill interference for $a=\sigma$, i.e., $f_{\text {int }}^{a=\sigma}=0$. Indeed, the braiding process where the edge Ising anyon encircles the bulk Ising anyon nontrivially rotates the system's quantum state such that $\left|\phi_{0}^{a}\right\rangle$ and $\left|\phi_{1}^{a}\right\rangle$ become orthogonal (similar to electrical non-Abelian FQH interferometry $[10,11])$. Interference can, however, survive for $a=\mathbb{1}, \psi$-provided $s_{2}$ also retrieves energy $\sim 2 h_{z}$ in the paths from Fig. 2(b). In these $\mathcal{O}\left(\tilde{t}_{\sigma}\right)$ paths, energy partitions between the edge Ising anyons in all ways compatible with energy conservation (see Supplemental Material [33]), ensuring a finite energy-retrieval probability even when the Ising-anyon wave packets in Fig. 2(b) are well-separated. The interference corrections for $a=\mathbb{1}, \psi$ satisfy $f_{\text {int }}^{a=\psi}=-f_{\text {int }}^{a=1}$, where the minus sign reflects the Abelian statistical phase acquired when the edge Ising anyon 
encircles a bulk fermion. Thus, $s_{2}^{z}$ measurements distinguish all three bulk quasiparticle types as summarized in Fig. 2(c).

For a quantitative treatment, we take $a=\mathbb{1}$ and evaluate the $\mathcal{O}\left(\Lambda_{1}^{2} \Lambda_{2}^{2} \tilde{t}_{\sigma}\right)$ energy-shuttling interference correction to $\left\langle s_{2}^{z}(t)\right\rangle$ at late times $t \gg t_{2}$. The trivial path corresponds to

$$
\begin{aligned}
\left|\phi_{0}^{a=1}\left(t \gg t_{2}\right)\right\rangle \approx & -e^{i \frac{2 h_{z}}{v}\left(x_{2}-x_{1}\right)} \frac{1}{6 \sqrt{\pi}} \Lambda_{1} \Lambda_{2}\left(h_{z} \tau\right) \\
& \times|0\rangle \otimes\left|s_{1}^{z}=\downarrow, s_{2}^{z}=\uparrow\right\rangle+\cdots,
\end{aligned}
$$

where we displayed only the term describing energy shuttling from $s_{1}$ to $s_{2}$ that is relevant for interference. Overlap with the nontrivial energy-shuttling paths encoded by $\left|\phi_{1}^{a=\mathbb{1}}\left(t \gg t_{2}\right)\right\rangle$ thus follows from

$$
\begin{aligned}
\langle 0| \otimes & \left\langle\downarrow, \uparrow \mid \phi_{1}^{a=\mathbb{1}}\right\rangle \\
= & i \int_{t_{a}, t_{b}, t_{c}} \frac{\lambda_{1}\left(t_{a}\right) \lambda_{2}\left(t_{b}\right)}{(2 \pi)^{2}} e^{2 i h_{z}\left(t_{b}-t_{a}\right)} \\
& \times\left\langle 0\left|T\left(t_{b}, x_{2}\right)\left[H_{\mathrm{tun}}\left(t_{c}\right)-\left\langle H_{\mathrm{tun}}\left(t_{c}\right)\right\rangle\right] T\left(t_{a}, x_{1}\right)\right| 0\right\rangle .
\end{aligned}
$$

The $\left\langle H_{\text {tun }}\right\rangle$ term simply compensates the correction to the vacuum state due to Ising-anyon tunneling. Supplemental Material [33] evaluates the conformal field theory correlator in Eq. (6) and in the late-time limit obtains

$$
\begin{aligned}
f_{\text {int }}^{a=\mathbb{1}}\left(t \gg t_{2}\right) \approx & -\frac{\tilde{t}_{\sigma}}{192}\left(\Lambda_{1} \Lambda_{2}\right)^{2}\left(\frac{v \tau}{L_{a}}\right)\left(h_{z} \tau\right) \sin \left(2 h_{z} L_{a}\right) \\
& \times e^{-\frac{\Delta^{2}}{(2 v \tau)^{2}}}\left[e^{-\frac{\left(\Delta+L_{a}\right)^{2}}{(2 v \tau)^{2}}}-e^{-\frac{\left(\Delta-L_{a}\right)^{2}}{(2 v \tau)^{2}}}\right] .
\end{aligned}
$$

Supplemental Material [33] further derives the correction from interference between the $\mathcal{O}\left(\tilde{t}_{\sigma}\right)$ energy-shuttling paths and the precession of both spins-which exhibits oscillatory dependence on the measurement time $t$.

Several comments are warranted. After energy retrieval by $s_{2}$, the edge returns to the vacuum state in the Fig. 2(a) path but retains two Ising-anyon wave packets separated by a distance $L_{a}$ in the $\mathcal{O}\left(\tilde{t}_{\sigma}\right)$ Fig. 2(b) paths. Consequently, the late-time interference correction is proportional to $\left\langle 0\left|\sigma(x) \sigma\left(x+L_{a}\right)\right| 0\right\rangle \sim L_{a}^{-1 / 8} \sim \tilde{t}_{\sigma} / L_{a}$, explaining the power-law $L_{a}$ dependence in Eq. (7). The two bracketed exponentials in Eq. (7) correspond to the processes where $s_{2}$ absorbs energy $\sim 2 h_{z}$ from the tunneled Ising anyon in Fig. 2(b). These paths accumulate a dynamical phase $\pm 2 h_{z} L_{a}$ relative to Fig. 2(a) due to the path-length difference-hence the sin factor in Eq. (7). [The Ising-anyon energy packet that remains coincident with the trivial path acquires no relative phase, and thus its absorption by $s_{2}$ does not contribute to interference between Figs. 2(a) and 2(b).] For wide pulses with $v \tau \gtrsim L_{a}, \lambda_{2}(t)$ temporally overlaps with both edge Ising anyons and enables $\boldsymbol{s}_{2}$ to draw energy from both the advanced and delayed packets.
The resulting interference signal is maximized when the pulse width and interference path length are comparable $\left(v \tau \sim L_{a}\right)$ and when the timing favors one of the tunneling paths $\left(\Delta \sim \pm L_{a}\right)$. If the ancillary-spin Zeeman splitting can be controlled, the oscillatory $h_{z}$ dependence provides an additional interferometric signature. For very narrow pulses such that $v \tau \ll L_{a}, \lambda_{2}(t)$ cannot have appreciable temporal overlap with the arrival of energy both from the trivial path and from one of the displaced Ising-anyon packets. Under such conditions interference instead arises from the process where both spins precess in the Zeeman field, which allows for overlap between $\lambda_{2}(t)$ and the splintered energy packet for timing $\Delta=0, \pm L_{a}$ (see Supplemental Material [33]).

Fermions can also tunnel across the constriction, adding a less-relevant $-i t_{\gamma} \gamma\left(x_{b}\right) \gamma\left(x_{t}\right)$ term to Eq. (3). At $\mathcal{O}\left(t_{\psi}\right)$, paths analogous to Fig. 2(b) arise wherein the incident energy splinters into displaced fermion wave packets. These paths underlie similar interference corrections to $\left\langle s_{2}^{z}(t)\right\rangle$ but with different sensitivity to bulk quasiparticles: The $\mathcal{O}\left(t_{\psi}\right)$ interference correction $g_{\text {int }}^{a}$ satisfies $g_{\text {int }}^{a=\mathbb{1}}=$ $g_{\text {int }}^{a=\psi}=-g_{\text {int }}^{a=\sigma}$. The first equality arises because a fermion encircling either a boson or fermion yields a trivial statistical phase; the minus sign in the second equality reflects the -1 statistical phase acquired when a fermion encircles an Ising anyon. Fermion tunneling thus shifts the late-time probabilities in Fig. 2(c), but, importantly, all three quasiparticle types generically remain distinguishable.

Discussion. - Our theory implicitly assumes that the spin liquid's bulk excitation gap exceeds the ancillary-spin Zeeman energy so that the gapless edge mode provides the dominant energy-shuttling medium. Given the $\mathcal{O}(10 \mathrm{~T})$ field required to reach the purported non-Abelian spin-liquid phase in $\alpha-\mathrm{RuCl}_{3}$, ancillary spins with spin orbit suppressed and/or tunable $g$ factors (as in, e.g., Refs. [37-39]) are desirable both to satisfy this constraint and for probing the oscillatory interference correction in Eq. (7). We stress, however, that physical spins may be substituted for arbitrary addressable quantum two-level systems-quantum dots, defect centers, etc. - that can locally couple to the edge, opening the door to a broader set of techniques for tuning the ancilla level splitting such as the ac Stark effect.

At finite temperature, edge-phonon coupling-which can be important for thermal transport [40,41] —allows injected energy to leak into the bulk, even when the spinliquid gap is "large." We argue that phonon-transport corrections play a minor role in our context for two reasons: (i) Whereas the edge mode serves as an energy waveguide between the ancillary spins, energy carried by phonons has a relatively low probability of reaching the absorber spin $\boldsymbol{s}_{2}$. (ii) Phonons that do reach $s_{2}$ will generally not arrive simultaneously with edge wave packets; timing considerations thus further suppress the impact of phonon-mediated energy shuttling. By considering the relative velocity of (slow) bulk phonons and the (fast) edge mode, one can, moreover, optimize the relative separation of the ancilla to 
minimize the phonon contribution. Such corrections might be further suppressed by introducing geometric constraints such as a narrow channel between the ancilla that reduces the bulk phonon conductance but without being so narrow as to introduce unwanted interedge tunneling. Phonon leakage can still reduce the energy-absorption "kick" for well-timed pulses [Figs. 1(b) and 2(c)] but is not expected to qualitatively alter our conclusions.

Edge-state interrogation does not require phase coherence and thus provides an enticing preliminary experiment. For time-domain anyon interferometry, by contrast, the edge distance $x_{2}-x_{1}$ between the ancillary spins must be sufficiently small that phase coherence persists. Moreover, the $\lambda_{j}(t)$ timescale $\tau$ should satisfy $h_{z} \tau \gg 1$ to maintain approximate energy conservation along with $L_{\text {perimeter }}>v \tau$ ( $L_{\text {perimeter }}$ is the spin liquid's total perimeter) to avoid trivial self-interference of pulses. To get a very rough sense of scales, if $h_{z} \sim 1 \mathrm{~K}, v \sim 10^{4} \mathrm{~m} / \mathrm{s}$, and $L_{\text {perimeter }} \sim 10 \mu \mathrm{m}$, then these conditions are satisfied for $\tau \sim 1 \mathrm{~ns}$. When $h_{x}=0$ so interference comes strictly from Eq. (7), we further desire that $v \tau \sim L_{a}$; with $L_{a} \sim 1 \mu \mathrm{m}$, this condition holds for $\tau \sim 0.1 \mathrm{~ns}$. For these parameters, the edgeshuttling and interferometry contributions remain perturbative but nonnegligible for an edge-ancilla coupling $\Lambda \sim 1$. If we aim only to probe the chiral edge mode, then the range of useful couplings increases significantly, as $\Lambda$ can be boosted by increasing the pulse width $\tau$ or by performing a series of pulses.

We expect that temporal control over $\lambda_{j}(t)$ can be substituted by a time-dependent Zeeman energy (or other qubit splitting) as has been explored in the context of Levitons and FQH systems [29,42]. More generally, timedomain anyon interferometry naturally adapts to other chiral topological phases where electrical transport measurements are challenging (Mott insulators, cold atoms, etc.). The background magnetic field in this more general context need not be "large"-potentially eschewing the issue of suppressing the ancilla level splitting. And finally, the fractionalized excitations need not be non-Abelian; single-anyon detection requires only relative phase accumulation associated with braiding around a quasiparticle.

We are grateful to Gabor Halasz, Erik Henriksen, Jason Petta, Ady Stern, and Ashvin Vishwanath for illuminating discussions. This work was supported by the Army Research Office under Grant No. W911NF17-1-0323; the National Science Foundation through Grants No. DMR-1723367 and No. DMR-1848336; the Caltech Institute for Quantum Information and Matter, an NSF Physics Frontiers Center with support of the Gordon and Betty Moore Foundation through Grant No. GBMF1250; the Harvard-MIT CUA, ARO Grant No. W911NF-20-10163; the AFOSR-MURI Photonic Quantum Matter Grant No. FA95501610323; and the Walter Burke Institute for Theoretical Physics at Caltech. The final stage of this work was in part based on support by the U.S. Department of Energy, Office of Science through the Quantum Science Center (QSC), a National Quantum Information Science Research Center.

[1] A. Y. Kitaev, Ann. Phys. (Amsterdam) 303, 2 (2003).

[2] C. Nayak, S. H. Simon, A. Stern, M. Freedman, and S. Das Sarma, Rev. Mod. Phys. 80, 1083 (2008).

[3] R. Willett, J. P. Eisenstein, H. L. Störmer, D. C. Tsui, A. C. Gossard, and J. H. English, Phys. Rev. Lett. 59, 1776 (1987).

[4] G. Moore and N. Read, Nucl. Phys. B360, 362 (1991).

[5] M. Levin, B. I. Halperin, and B. Rosenow, Phys. Rev. Lett. 99, 236806 (2007).

[6] S.-S. Lee, S. Ryu, C. Nayak, and M. P. A. Fisher, Phys. Rev. Lett. 99, 236807 (2007).

[7] D. T. Son, Phys. Rev. X 5, 031027 (2015).

[8] M. Banerjee, M. Heiblum, V. Umansky, D. E. Feldman, Y. Oreg, and A. Stern, Nature (London) 559, 205 (2018).

[9] S. Das Sarma, M. Freedman, and C. Nayak, Phys. Rev. Lett. 94, 166802 (2005).

[10] A. Stern and B. I. Halperin, Phys. Rev. Lett. 96, 016802 (2006).

[11] P. Bonderson, A. Kitaev, and K. Shtengel, Phys. Rev. Lett. 96, 016803 (2006).

[12] W. Bishara and C. Nayak, Phys. Rev. B 77, 165302 (2008).

[13] R. L. Willett, K. Shtengel, C. Nayak, L. N. Pfeiffer, Y. J. Chung, M. L. Peabody, K. W. Baldwin, and K. W. West, arXiv:1905.10248.

[14] J. Nakamura, S. Liang, G. C. Gardner, and M. J. Manfra, arXiv:2006.14115.

[15] A. Kitaev, Ann. Phys. (Amsterdam) 321, 2 (2006).

[16] G. Jackeli and G. Khaliullin, Phys. Rev. Lett. 102, 017205 (2009).

[17] S. Trebst, arXiv:1701.07056.

[18] Y. Kasahara, T. Ohnishi, Y. Mizukami, O. Tanaka, S. Ma, K. Sugii, N. Kurita, H. Tanaka, J. Nasu, Y. Motome, T. Shibauchi, and Y. Matsuda, Nature (London) 559, 227 (2018).

[19] T. Yokoi, S. Ma, Y. Kasahara, S. Kasahara, T. Shibauchi, N. Kurita, H. Tanaka, J. Nasu, Y. Motome, C. Hickey, S. Trebst, and Y. Matsuda, arXiv:2001.01899.

[20] K. W. Plumb, J. P. Clancy, L. J. Sandilands, V. V. Shankar, Y. F. Hu, K. S. Burch, H.-Y. Kee, and Y.-J. Kim, Phys. Rev. B 90, 041112(R) (2014).

[21] L. E. Chern, E. Z. Zhang, and Y. B. Kim, Phys. Rev. Lett. 126, 147201 (2021).

[22] D. Aasen, R. S. K. Mong, B. M. Hunt, D. Mandrus, and J. Alicea, Phys. Rev. X 10, 031014 (2020).

[23] Y.-H. Zhang and A. Vishwanath, arXiv:2005.12925.

[24] J. Feldmeier, W. Natori, M. Knap, and J. Knolle, Phys. Rev. B 102, 134423 (2020).

[25] E. J. Konig, M. T. Randeria, and B. Jack, Phys. Rev. Lett. 125, 267206 (2020).

[26] R. G. Pereira and R. Egger, Phys. Rev. Lett. 125, 227202 (2020).

[27] M. Udagawa, S. Takayoshi, and T. Oka, Phys. Rev. Lett. 126, 127201 (2021). 
[28] R. C. Ashoori, H. L. Stormer, L. N. Pfeiffer, K. W. Baldwin, and K. West, Phys. Rev. B 45, 3894 (1992).

[29] J. Keeling, A. V. Shytov, and L. S. Levitov, Phys. Rev. Lett. 101, 196404 (2008).

[30] N. Yao, C. Laumann, A. Gorshkov, H. Weimer, L. Jiang, J. Cirac, P. Zoller, and M. Lukin, Nat. Commun. 4, 1585 (2013).

[31] P. L. S. Lopes, I. Affleck, and E. Sela, Phys. Rev. B 101, 085141 (2020).

[32] Any pulse shape $\lambda_{j}(t)$ suffices provided it becomes negligible after some finite time and varies sufficiently smoothly.

[33] See Supplemental Material at http://link.aps.org/ supplemental/10.1103/PhysRevLett.126.177204 for a detailed derivation of the results presented in the main text, including both the perturbative calculation of $\left\langle s_{2}^{z}(t)\right\rangle$, and a conformal field theory treatment of the Ising anyon tunneling.

[34] P. Fendley, M. P. A. Fisher, and C. Nayak, Ann. Phys. (Amsterdam) 324, 1547 (2009), July 2009 Special Issue.
[35] P. Fendley, M. P. A. Fisher, and C. Nayak, Phys. Rev. B 75, 045317 (2007).

[36] P. Fendley, M. P. A. Fisher, and C. Nayak, Phys. Rev. Lett. 97, 036801 (2006).

[37] M. F. Doty, M. Scheibner, I. V. Ponomarev, E. A. Stinaff, A. S. Bracker, V. L. Korenev, T. L. Reinecke, and D. Gammon, Phys. Rev. Lett. 97, 197202 (2006).

[38] J. H. Prechtel, F. Maier, J. Houel, A. V. Kuhlmann, A. Ludwig, A. D. Wieck, D. Loss, and R. J. Warburton, Phys. Rev. B 91, 165304 (2015).

[39] S. Studenikin, M. Korkusinski, M. Takahashi, J. Ducatel, A. Padawer-Blatt, A. Bogan, D. G. Austing, L. Gaudreau, P. Zawadzki, A. Sachrajda, Y. Hirayama, L. Tracy, J. Reno, and T. Hargett, Commun. Phys. 2, 159 (2019).

[40] M. Ye, G. B. Halsz, L. Savary, and L. Balents, Phys. Rev. Lett. 121, 147201 (2018).

[41] Y. Vinkler-Aviv and A. Rosch, Phys. Rev. X 8, 031032 (2018).

[42] G. Wagner, D. X. Nguyen, D. L. Kovrizhin, and S. H. Simon, Phys. Rev. B 100, 245111 (2019). 